\title{
Replicating our Instructional Capacity: Developing Shared Workshops across Institutions
}

Danica Lewis, Collections \& Research Librarian for Life Sciences, NCSU

Alex Carroll, Research Librarian for Engineering and Biotechnology, NCSU

Barrie Hayes, Bioinformatics and Research Data Librarian, UNC-CH 


\section{Molecular Biology Searching, Briefly}

What makes this such a challenging workshop topic for librarians? 
BLAST ${ }^{\circ}$ " blastn suite

\section{Standard Nucleotide BLAST}

$\sqrt{\text { blastn }}$ blastp $\mid$ blastx $\mid$ thlastn $\mid$ thlastx

Enter Query Sequence

Clear

Query subrange \&

TCAGATAGGGGTCCCTTGACCACCATCCTCCGTGAAATCAATATCCCGCACAAGAGTGCTACT

TCAGATAGGG

From

CTCCGGGCCCATAACACTTGGGGGTAGCTAAAGTGAACTGTATCCGACATCTGGTTCCTACT

TCAGGGCC

ATAAAGCCTAAATAGCCCACACGTTCCCCTTAAATAAGACATCACGATG

To
Or, upload file
Choose file no flio seloctoo
9
Job Title
Enter a descriptive titse for your BLAST search of

Align two or more sequences 8

Choose Search Set

Database OHuman genomic + transcript Mouse genomic + transcript COthers (nr etc):

- Human genomic plus transcript (Otuman G+T) D (176974 sequences) $\theta$

Exclude $\square$ Models $(X M X P) \square$ Uncultured/environmental sample sequences

Optional

Limit to

Entrez Query

Optional

Sequences from type material

Enter an Entrez query to limit search 8

You Lifbe Create custom database

Program Selection

Optimize for

- Highly similar sequences (megablast)

More dissimilar sequences (discontiguous megablast)

Somewhat similar sequences (blastn)

Choose a BLAST algorithm \&

BLAST Search database Human G+T using Megablast (Optimize for highly similar sequences)

Show results in a new window 


\section{BLAST ${ }^{\circledR}$ " blastn suite » RID-HPPZOPCV013}

Edit and Resubmit Save Search Strategies $\triangleright$ Eormatting options $\gg$ Download Job title: Nucleotide Sequence (16569 letters)

RID HPPZOPCVO13 (Expires on 05-18 04:06 am)

Query ID Id|Query_213677

Description None

Molecule type nucleic acid

Query Length 16569

Other reports: $\triangleright$ Search Summary [Taxonomy reports] [Distance tree of results] [Genome view] [MSA viewer]

Eraphic Summary

Distribution of the top 169 Blast Hits on 69 subject sequences

Mouse over to see the title, click to show alignments

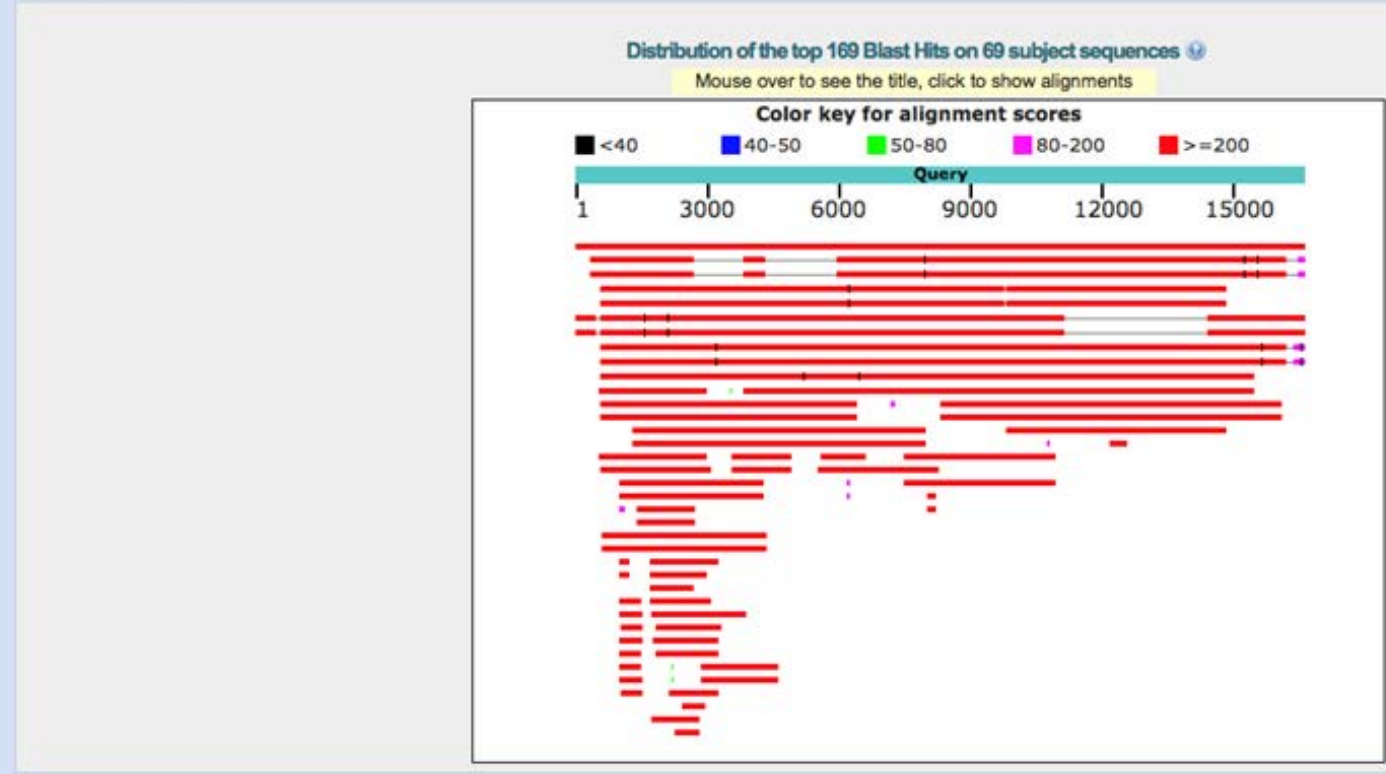

Yeu fillite How to read this page Blast report description

Database Name Human G+T (2 databases)

Description $\triangleright$ See details

Program BLASTN 2.6.1 $+\triangleright$ Citation 


\section{Our Communities Want Training On This!}

Poultry Science

Population Health \& Pathobiology

Molecular Biomedical Sciences

Microbiology

Marine Sciences

Genomics

Food Science

Animal Science

Entomology
Environmental Science

Chemical \& Biomolecular Engineering

Medicine

Biological Sciences

Biochemistry \& Biophysics

Pharmacy

Public Health

Applied Statistics

Dentistry 


\section{Why Recycling Old Content Doesn't Work}

How many of you have experienced the following:

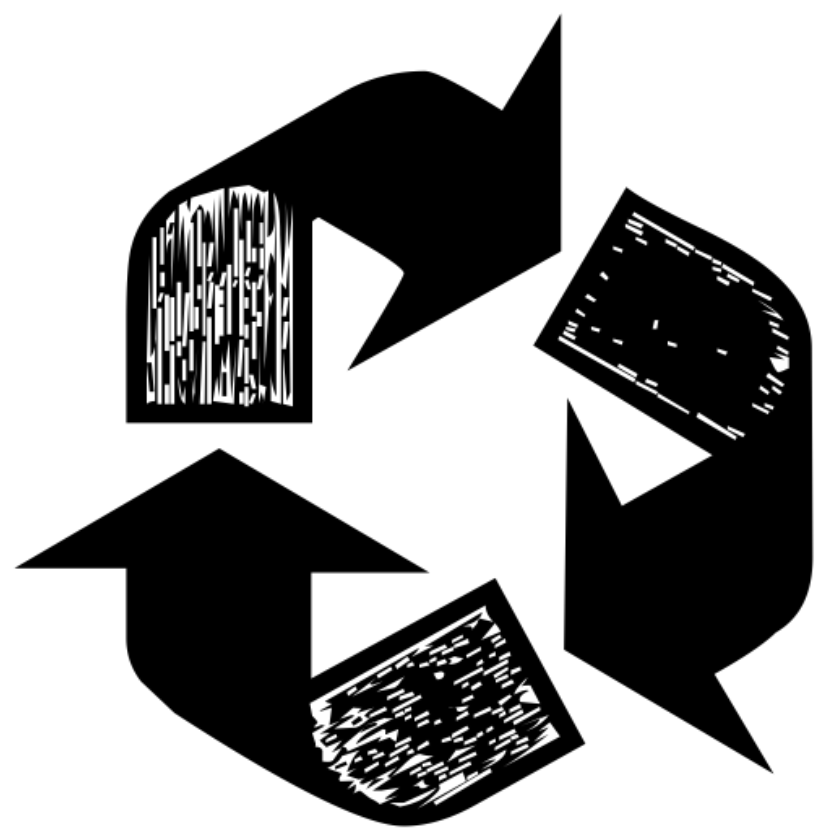




\section{Our Collaborative Workshop Development Workflow}

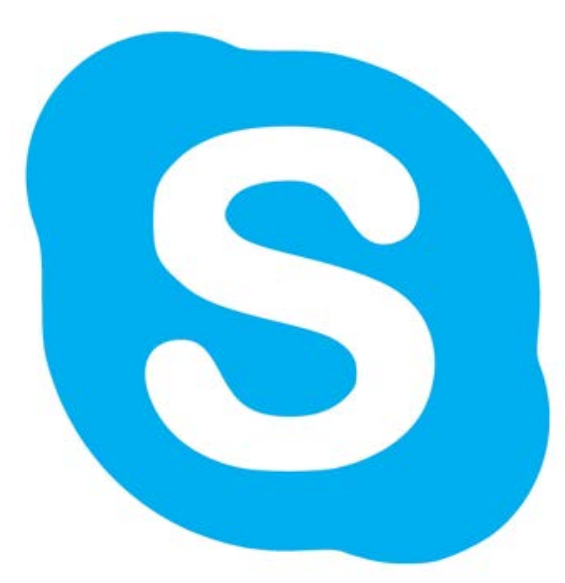

Video Conferencing

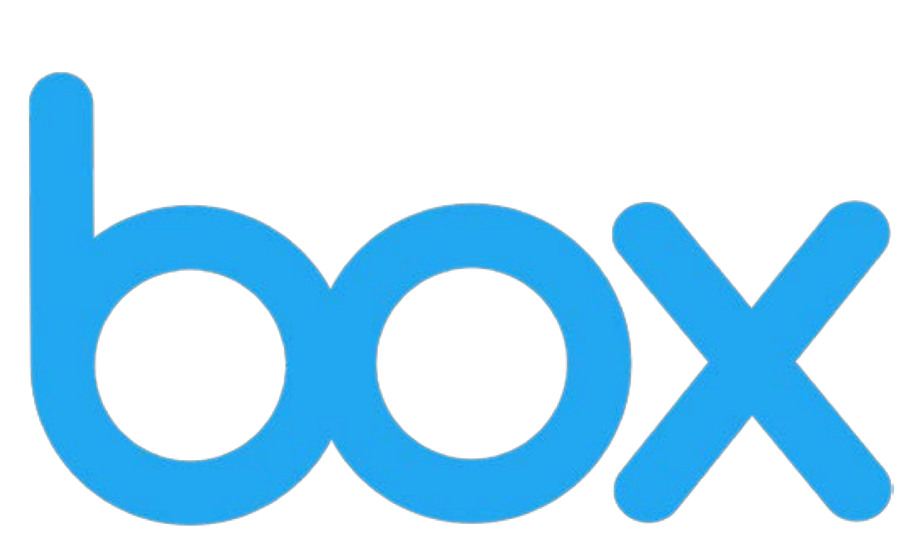

$\rightarrow$ Cloud Storage

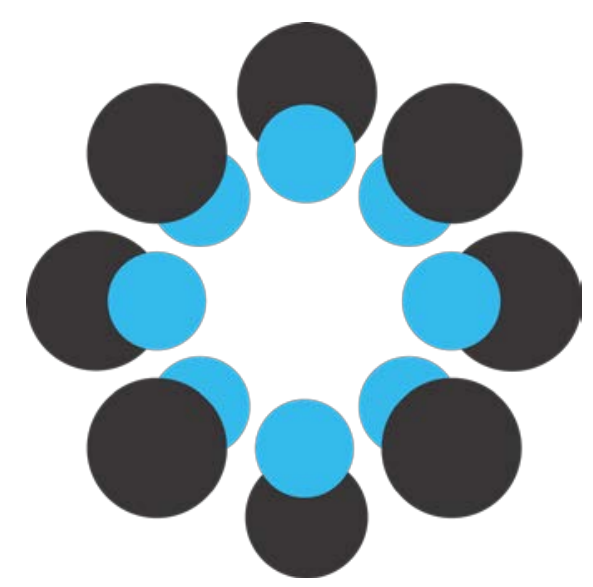

Open Repository with Version Control 


\section{Benefits of this Approach}

- Division of Labor

- Community of Practice

- Accountability

- Increased Reach

- Giving Back to the Broader Profession 


\section{Possible Areas for Collaborative Workshops}

Research Impact \& Bibliometrics

Open Scholarship \& Authors' Rights

Open Education

GIS

Online Scholarly Identity (ORCID, etc.)

Coding \& Programming

Data Visualization (Tableau, Excel)
Citation Management

Open Data Repositories (GitHub, OSF)

Science Communication

Data Management Planning

Digital Media Production

Informed Consent, IRB/IACUC

Makerspaces \& Design Thinking 
Check Out our Workshops!

\section{https://osf.io/a6x2q/}




\section{Questions?}

Danica Lewis: dmlewis6@ncsu.edu

Alex Carroll: ajcarro4@ncsu.edu

Barrie Hayes: bhayes@email.unc.edu 